\title{
Genetics of Attention Deficit Hyperactivity Disorder (ADHD): Recent Updates and Future Prospects
}

\author{
Russell Schachar
}

Published online: 30 January 2014

(C) Springer International Publishing AG 2013

\begin{abstract}
Attention deficit hyperactivity disorder (ADHD) is not only highly prevalent, persistent and impairing but also is one of the most heritable of all psychiatric disorders. As a result, ADHD has been the focus of considerable genetic research. The results of recent genetic studies are reviewed with a focus on the emerging picture and future trends. ADHD appears to be a complex disorder in which multiple genetic and environmental risks contribute to a quantitative trait. At the same time, there is growing evidence that in a proportion of cases, individually rare variants such as copy number variants may play an important causal role. The more genetic risks, both common and rare, the more extreme the trait. With increasing samples and advanced genetic methods, single nucleotide polymorphism (SNPs) and copy number variants $(\mathrm{CNVs})$ conferring risk for ADHD are being identified. Further study will be required before we can understand the causal significance of these findings. Increased sample size is an urgent necessity if we are to discover potentially causal variants. Non-behavioral markers of genetic risk known as endophenotypes could also play a role in parsing the phenotypic and genetic heterogeneity of ADHD as they have in other complex disorders. Genetic studies in ADHD hold the potential for refined nosology, more precise diagnosis, and differential diagnosis, improved early identification leading to novel intervention strategies and identification of innovative targets for therapeutics based on a precise understanding of disease mechanism.
\end{abstract}

Keywords Attention deficit hyperactivity disorder . Genetics · Endophenotypes · Candidate genes · Genome-wide · GWAS · CNV · Twin study · Family study

R. Schachar $(\bowtie)$

The Hospital for Sick Children, University of Toronto, Toronto, ON, Canada

e-mail: russell.schachar@sickkids.ca

\section{Introduction}

Attention deficit hyperactivity disorder (ADHD) has been the focus of considerable genetic research over the last few years. Since the initial family and twin studies of the early 1970s [1, 2], there have been about 2,800 publications on ADHD and genetics with over 300 appearing in 2012-2013 alone. These publications include numerous family and twin studies, 24 genome-wide association studies, and 32 meta-analyses of genetic findings exploring the genetic architecture of ADHD. These studies support the role of genetic influences on ADHD (HugeNavigator; www.cdc. gov/genomics/hugenet/hugenavigator.htm).

ADHD tends to be a controversial disorder for various reasons. It has a very high prevalence which varies widely from $2-3 \%$ to $10 \%$ depending on the assessment measures that are used, the population one is studying, and the specific diagnostic criteria and thresholds that are applied (in particular criteria for pervasiveness of symptoms and impairment)[3]. The diagnosis is applied to those at the extreme of a continuously distributed trait without a clear-cut and universally agreed threshold [4], and is measured indirectly using parents or teachers as informants rather than directly with a laboratory test (there are no known biomarkers or tests for ADHD). Disagreements among informants are common and are taken to reflect the subjectivity of the trait, its situational specificity or measurement error. Many cases remit although most continue. Many individuals with an ADHD diagnosis exhibit other disorders (comorbidity) raising the possibility that ADHD could be a non-specific consequence or epiphenomenon of other disorders rather than a specific disorder. The most common comorbid conditions are conduct disorder (30\%), depression (30\%), anxiety disorder (20\%), learning disabilities $(50 \%)$, and, in a smaller number of cases, bipolar disorder, autism spectrum disorder (ASD) and eating disorders [5]. In a few instances, ADHD presents in the context of a 
neurodevelopmental disorder such as one of the rare deletion syndromes like velo-cardio-facial syndrome (VCF, 22q11 deletion). Numerous biological, psychological and social factors such as prenatal exposure to alcohol, traumatic brain injury $[6,7]$, prematurity, low birth weight $[8]$, treatment for leukemia and psychosocial adversity increase the risk for ADHD. Even current treatments for ADHD are controversial because they may have limited long-term benefit.

Although this picture of complexity and uncertainty is identical to that found in other disorders including most medical conditions, many use it to challenge the existence of ADHD. Genetics offers the promise of more precise diagnosis, refinement of current nosology, early detection of affected individuals, prediction of outcome, prevention, and eventually both improved and increasingly specific treatments [9]. This review focuses on the most recent developments in the genetics of ADHD, sets the scene for future developments, and discusses implications of current genetic discoveries for clinical practice.

The picture of ADHD that is emerging is one of a "complex" disorder much like heart disease or obesity and similar to other psychiatric disorders such as schizophrenia, autism spectrum disorder (ASD) and bipolar disorder. There are two genetic models of ADHD driving current research. The common disease - common variant model of ADHD postulates that multiple genetic and environmental influences each contribute a small amount to a disease phenotype. The greater the number of these influences, the more extreme the observable phenotype. Proximally, genetic variants influence synthesis, structure and function of proteins and distally perturb brain structure giving rise to variation in physiology, cognition (thinking, feeling, and acting), and other indices of neuronal dysfunction which, in turn, engender variations in the observable (phenotypic) manifestations of the disorder. Typically, phenotype refers to observable phenomena associated with disease even though any variation that is not genetic, strictly speaking, is a phenotype. In complex disorders, environmental factors play an important role by influencing gene expression, by shaping the pathway from gene to phenotype or directly impacting on disease expression. This model is consistent with the fact that restlessness, inattentiveness and impulsiveness vary widely in the general population and generally follow a normal, bell-shaped distribution. ADHD is a diagnosis that practitioners apply when these behaviors reach a level that is extreme for the population (DSM IV and DSM 5)[10, 11]. By contrast, a rare variant model postulates that there are many individual genetic variants any one of which could cause ADHD or contribute a great deal to the phenotype in a particular individual. A single causal variant could be limited to only one family or to very few families in the population. Other rare variants might cause the same disorder in other individuals. Some cases might be genetic but not inherited because the abnormal gene or genes arises de novo. For the most part it appears that both common and rare variants are likely to play a role in ADHD and in other mental illnesses $[12,13 \bullet \bullet]$.

\section{Family Studies of ADHD}

Practitioners have for some time observed that ADHD "runs in families" suggesting, but not proving, that it could be influenced by a person's genes. The distribution of a trait within families has been used to reveal the influence of genetic factors on that trait because family members share a predictable portion of their inherited genetic variation as a function of the degree of their relatedness. For example, siblings share on average $50 \%$ of genetic variation (identical by descent) and cousins share $25 \%$ whereas the extent of genetic relatedness among two individuals from the general population is far lower. Also, families are more homogeneous in genetic background and may be more similar in their exposure to environmental risks than are unrelated cases. If a person is unrelated to an individual with ADHD, their risk of having ADHD ought to be like that of anyone in the general population, namely 5$10 \%$. But if a person is related, e.g., a sibling of an individual with a diagnosis of ADHD, who shares $50 \%$ of genetic inheritance on average, he or she ought to be at increased risk. This prediction has been confirmed in numerous family studies: The risk to first-degree relatives of ADHD individuals is 15-60\% (2-6 relative risk) [14] confirming clinical observations and demonstrating genetic influence on ADHD.

In addition to demonstrating the familial nature of ADHD, family studies play a role in the validation of ADHD comorbid subtypes. If comorbidity delineates a genetically distinct subtype of ADHD, one should see that particular subtype clustering (breeding true, cosegregating) among relatives within families. By contrast, if a comorbid condition is a variable manifestation of common genetic risks (pleiotropy), one would not expect that a comorbid condition would breed true within families. Family risk studies indicate that ADHD with comorbid conduct disorder (CD) [15], pediatric-onset bipolar [16] disorder or ASD [17] tend to cosegregate in families and, therefore, could represent potentially distinct genetic subtypes. By contrast, similar designs have not revealed cosegregation between nicotine dependence and ADHD [18].

\section{Twin Studies of ADHD}

Although family studies provide clear evidence for genetic influence on a trait, they do not allow for an estimate of the heritability of that trait because family members also share an unspecified amount of environmental influences. Heritability is the proportion of phenotypic variation due to additive genetic factors. For the most part, heritability has been estimated from twin studies, which allow for estimation of genetic 
and environmental influences on a trait. Twin studies capitalize on the fact that monozygotic (MZ; identical twins) share $100 \%$ of genetic inheritance whereas dizygotic (DZ; nonidentical twins) share on average $50 \%$ of their genetic inheritance. If one assumes for the moment that $\mathrm{MZ}$ and $\mathrm{DZ}$ twins share common environments to a comparable extent, the degree to which genetic factors influence a trait or disease will be evident in the extent to which MZ twins are more alike than DZ twins. Twin models partition covariance between MZ and DZ twins into additive genetic influences on a trait (A), common environmental influences $(\mathrm{C}$, those that make twins more alike) and non-shared or unique environmental influences (E, those that make twins less alike). Heritability $\left(h^{2}\right)$ is a number that reflects the proportion of variation in a trait among individuals in a population that is due to their genotypes.

There have been numerous twin studies of ADHD demonstrating high heritability of ADHD ( $\sim 75 \%)$ regardless of whether ADHD it is measured as a disorder (affected versus unaffected), a trait (more or less restless, inattentive and impulsive), as the extreme of a trait distribution, and by either parent or teacher ratings [19]. Typically, twin studies find little in the way of shared environmental effects [20 $]$ although twin studies tend to underestimate the contribution of environmental and over-estimate the extent of genetic risk factors. ADHD heritability estimates are as high as estimates for other biological traits such as height and as high as estimates for other mental illness such as ASD and schizophrenia, but these later disorders are far less prevalent than ADHD. Therefore, one could argue that ADHD is the most important genetically influenced mental illness of children and youth $[21 \bullet \bullet]$.

Recently, twin studies have been exploited to address important issues in the nature of ADHD beyond the question of heritability. Analyses of longitudinal twin data show that there are genetic influences that are stable thereby affecting ADHD traits throughout childhood and into adolescence, genetic influences that emerge with time and those that influence early development but attenuate with development [22]. Moreover, the longitudinal relationship between the ADHD dimensions appears to be unidirectional, with hyperactivity-impulsivity in middle childhood predicting the presence of inattentiveness in early adolescence, but not vice versa [23].

Twin studies can be used to assess coheritability of multiple traits in order to determine whether two traits have common genetic influences, a process known as pleiotropy. Twin studies reveal correlated familial genetic liabilities contributing to the comorbidity of ADHD and other neurodevelopmental disorders including ASD [17, 24-26], ADHD and bipolar disorder [27], ADHD (in particular inattentive symptoms) and reading disorder [28, 29], ADHD and mood symptoms, conduct problems, alcohol and substance use [30-32].

Heritability estimates are specific to populations. Sex and age with heritability are typically lower in adults. A recent developmental twin study showed that the shared view of selfrated and informant-rated ADHD traits is highly heritable throughout life [22]. This result goes some way to explain why previous twin studies of ADHD in adults have generated lower $\mathrm{h}^{2}$ estimates than in children. Estimates of genetic influences on ADHD are also affected by the nature of the rating scales that are used to measure ADHD with more detailed questionnaires, teacher ratings and objective measures of ADHD traits such as actigraphs (movement detectors) yielding lower heritability estimates for overall clinician or parent ratings [19].

Twin studies have their limitations. MZ and DZ may not have equally common environments due to sharing of placental environments, for example, and twin analyses cannot easily accommodate the effect of interactions or correlations between genetic and environmental risks which to tend to inflate heritability estimates. Despite these limitations, high heritability of ADHD underscores the value of genetic research and bodes well for gene mapping. High heritability indicates that there is a strong correlation between the phenotype and genotype so that genetic loci that have an effect on the trait should be detected more easily. However, high heritability does not establish that a trait has a simple genetic architecture. Heritability could, instead, be a result of many loci contributing to the trait just as a trait with low heritability could be caused by a single causal locus [20 [. Moreover, twin studies cannot easily identify genetic interactions with environmental influences.

\section{Candidate Gene Studies}

Although family and twin studies demonstrate the role of genetic influences in ADHD, they do not provide any means of identifying the specific genetic risks. A candidate gene study, which involves what one might think of as an educated guess about the mechanism of a disease, is one strategy to investigate specific risks. The method involves assessing the association of a particular allele or set of alleles of a gene and the disease itself to determine if the alleles are found more often in affected than unaffected individuals or is transmitted along with the disease. But which genes should be studied? In many complex diseases, scientists have little idea of the underlying mechanism making selection of a candidate gene problematic and markedly raising the risk of false positive associations. However, ADHD is known to respond to stimulant medication which in turn is recognized to affect release and reuptake of neurotransmitters in particular dopamine. Therefore, it was natural to target genes implicated in these processes and in other neurotransmitter systems in initial candidate gene studies. Candidate gene studies have regularly yielded evidence for association with several genes involved in dopaminergic (DRD4, DRD5, DAT1), serotonergic (SLC6A3 and 5-HTT/SLC6A4, HTR1B serotonin receptor 
gene), noradrenergic (such as NET1/SLC6A2, ADRA2A, ADRA2C) and nicotinergic (CHRNA4) neurotransmission and receptor function as well as genes involved in neurotransmission and neuronal plasticity (SNAP25, CHRNA4, NMDA, BDNF, NGF, NTF3, NTF4/5, GDNF) [33].

However, candidate gene approaches are limited by the validity of our a priori knowledge of disease mechanism, which is partial at very best. Candidate gene studies run a substantial risk of generating false positives because of the large number of gene associations that are tested individually without correction for the total number of possible associations and the probability of bias toward publishing positive but not negative results. It is not surprising then that it has been uncommon for genetic associations arising candidate gene studies to be replicated in genome-wide association studies [34]. Consequently, single association studies must be considered tentative and thoroughly replicated [35]. Being a complex trait, ADHD is very probably influenced by a number of different genetic risks and that will make identification of the role of any single risk difficult in the absence of very large populations. Finally, candidate gene studies focus on particular genetic markers, which may not be the causal gene but are linked or inherited along with the causative gene as it is passed from generation to generation. Only a few candidate genes have been replicated in genome-wide studies. For these reasons, scientists have moved away from candidate gene studies of ADHD toward linkage and association studies, which are not biased on a priori assumptions of disease mechanism.

\section{Genome-wide Approaches}

Both linkage and association designs use regularly spaced, highly variable (i.e., polymorphic) DNA segments termed markers (single nucleotide polymorphisms, SNPs) that are spread throughout the genome and whose genomic location is known precisely. In exome sequencing, these markers are concentrated in exomes, the $1 \%$ of the genome that plays a role in protein coding, but which is thought to harbor the majority of disease-causing mutations. Having said that, genetic associations in other diseases have identified risks as socalled gene deserts, which are regions with no known proteincoding variants [36••]. In association studies, genomes of individuals with ADHD are compared with genomes of unaffected individuals (controls) in order to identify differences. Any SNP marker that is found in disordered individuals significantly more often than in unaffected individuals is said to be associated with the disease. In linkage studies, genomes of various members of the same family with the same disorder are compared for sharing. Regions that are shared among similarly affected family members are said to be linked to the disease. In both linkage and association studies, identified regions are studied further to work out what gene may be carrying the risk [37].

Linkage and association studies of ADHD have not shown any associations that pass a rigorous level for genome-wide significance for the most part suggesting that the effects of common risk variants are individually very small, that sample sizes have been far too small or that multiple genetic pathways might lead to apparently common phenotypic consequences (genetic heterogeneity) [37, 38]. The problem of small sample size is a serious one and has been tackled by formation of the Psychiatric Genomics Consortium (PGC; http://pgc.unc.edu) in 2007 and the ADHD Genetics Consortium. The PGC aims to undertake mega-analyses using individual-level genotype and phenotype data rather than meta-analyses which are based on summary data such as odds ratios. Building and maintaining the necessary databases and shaping the required collaboration have been great achievements. The PGC incorporates GWAS data from more than 19 countries and for several major mental illnesses including ADHD. In 2013, the PGC published the results of the largest genome-wide analysis of psychiatric disorders to date by examining GWAS data in five disorders (ADHD, ASD, major depression, bipolar disorder and schizophrenia) in 33,332 cases and 27,888 controls of European ancestry [36 $\bullet^{\circ}$. SNPs at four loci surpassed the cutoff for genome-wide significance $\left(p<5 \times 10^{8}\right)$ in the primary analysis: regions on chromosomes 3p21 and 10q24, and SNPs within two L-type voltage-gated calcium channel subunits, CACNA1C and CACNB2. Except for CACNA1C (which suggested an affect limited to BPD and SCZ), these SNPS fit a model in which all five disorders in the analysis were similarly affected. These results implicate a specific biological pathway-voltage-gated calcium-channel signaling - as a contributor to the pathogenesis of several psychiatric disorders, and support the potential of this pathway as a therapeutic target for psychiatric disease.

Cross-disorder studies are pushing the field to consider ways in which a single variant could confer risk for phenomenologically distinct disorder (pleiotropy). Although the PGC study is groundbreaking, the approach is most suited to identify common variants rather than rare mutations or copy number variants (to be discussed below). Moreover, the PGC analyses cannot yet control for diagnostic misclassification, heterogeneity or comorbidity. Finally, the ADHD sample in the PGC analysis was the smallest of all disorders $(1,947$ child, mother and father trios and 840 cases compared with, for example, over 9,000 cases for SCZ and MDD).

Genome-wide analyses set high levels for statistical significance because they involve a large number of statistical tests (millions depending on the density of the array used). However, rigorous levels for significance in genome-wide studies could obscure the role of genes that individually contribute only modestly to ADHD. Pathway analysis is an approach for grouping individual, low intensity, genetic findings into 
functional networks to determine whether they converge within particular biological pathways. A recent pathway analysis showed that the top hits from ADHD GWAS studies tend to convergence in a limited number of pathways: 45 of the 85 top-ranked candidate genes encode proteins that play a role in the process of neurite outgrowth [39]. This finding fits well with research indicating widely distributed neural abnormalities and developmental delays (reduced cortical thickness and volume reductions) in ADHD and a wide range of resulting cognitive deficits. These results, however, are limited by the inadequacy of our knowledge about gene function.

Genome-wide methods offer entirely new techniques for assessing heritability of a trait in populations of apparently unrelated individuals. Genetic markers spread throughout the genome such as those assessed by microarrays can be used to estimate relatedness between pairs of individuals, because the more that individuals are related, the greater the sharing of marker alleles. The extent of estimated relatedness can be correlated with the magnitude of phenotypic similarity enabling heritability to be estimated. These estimates are known as SNP heritability $\left(h_{\mathrm{SNP}}^{2}\right)$. Applying this method, Wray and the PGC [36••] estimated that the heritability of ADHD was 0.28 . This estimate is lower than twin estimates because it omits the contribution of some causal variants that are not associated with common SNPs in most arrays, and that of less common or rare causal variants. Nevertheless, this estimate is a lower bound estimate of heritability and is as high as that of other mental illnesses and traits such as body mass index, Alzheimer's disease and multiple sclerosis when measured by SNP heritability. The Wray et al., study confirmed the coheritability (shared genetic risks) of ADHD and MDD (0.32) but did not detect significant shared genetic risk with ASD, BPD or schizophrenia contrary to previous twin and family studies.

\section{Copy Number Variations (CNVs)}

Copy number variation (CNVs) is one possible source of genetic variation in human diseases. Copy number variants are segments of DNA of various sizes in which there is a deletion, insertion or duplication, abnormal position (translocations) or orientation (inversions) of a DNA segment involving many SNPs. CNVs are not visible under a microscope like chromosomal abnormalities but they are much larger than single nucleotide variants (SNV). Some CNVs play a role in normal variation whereas other CNVs may be disease-causing. CNVs are found in everyone's genomes where they account for more of the total variation in the human genome than do SNPs. CNVs can be inherited or be de novo mutations, can disrupt gene function much like a SNP, and can increase or decrease the dose of a gene product.
Typically, one searches for CNVs using genome-wide methods akin to GWAS, but instead of looking for variation in SNPs, one is looking for larger portions of atypical DNA. Once found, it can be difficult to establish their pathogenicity. The highest priority CNVs are those that are not found in healthy control data sets, appear in the DNA of many affected individuals but not in unaffected individuals, and/or have been associated previously with neurodevelopmental diseases of various kinds. Large CNVs may be more likely to affect gene function than are smaller ones. Using this approach to prioritization, we and others have found evidence for association of CNVs with ADHD: $9 \%$ of ADHD cases had a priority CNV of which about $1.7 \%$ were de novo rather than inherited [40•]. Several of these CNVs were found in multiple cases providing good candidates for future work. Many of the CNVs that have been found in ADHD are known to appear in other neurodevelopmental disorders such as intellectual deficiency and ASD. For that reason, we studied a sample of ASD participants for the ADHD-related CNVs and were able to find ASD cases with the same variants in about half of observed CNVs. This observation of overlapping CNV findings provides another piece of evidence for common genetic mechanisms in neurodevelopmental disorders in particular ADHD and ASD [41]. The strongest association with ADHD and ASD was deletions in ASTN2 and TRIM32, but we also found shared genes in CHCHD3, MACROD2, and the 16p11.2 region. In a subsequent and larger study, Williams et al. [42] found an enrichment of rare CNVs in ADHD compared to controls with large CNVs $(>500 \mathrm{~kb})$ showing the greatest enrichment (1.28-fold). CNVs identified in ADHD case subjects were significantly enriched for loci implicated in ASD and in schizophrenia. Duplications spanning the CHRNA7 gene at chromosome 15q13.3 were associated with ADHD in single-locus analysis and a duplication at 15q13.3 appeared to be associated with comorbid conduct disorder. These results provide support for a role of rare CNVs in ADHD and confirm the theory that many neurodevelopmental disorders may share common genetic determinants.

\section{Environmental Risks and Genetics}

Many individual environmental risks have been associated with ADHD including low birth weight, maternal smoking, extreme environmental deprivation, maternal stress and alcohol use. What is less clear is whether these environmental exposures play a direct causative role in ADHD, increase risk for ADHD only in the presence of some genetic risks (gene by environment interactions) or are actually correlated with the actual genetic risk. For example, it is possible that the increased risk for ADHD among offspring of mothers who drink is a result of in utero exposure to alcohol or a result of inheritance from mothers who themselves have ADHD traits 
are, therefore, more likely to drink during pregnancy. Recent research on these topics involves the use of genetically informed designs such as comparison of the risk to offspring who are genetically related or unrelated by virtue of assisted conception [43]. Another approach [44] examines outcomes in relationship to specific candidate genes (e.g., DRD4 and DAT1) and environmental exposures (toxins and prenatal smoke exposure). Collectively, these studies have not found conclusive evidence for gene by environment interactions in ADHD. There is a clear need for further research of this kind.

\section{Endophenotypes}

One of the more interesting current debates in ADHD genetics has been about the potential role of endophenotypes in genetic research. An endophenotype (from Gr 'endo' meaning 'within' and 'pheinen' meaning 'to show') is a measure that reflects the action of genetic risks for disease, is biologically closer to the actual action of those genes and mediates the link between underlying genetic risk and phenotypic manifestations of disease. Cholesterol level has been used as an endophenotype in the study of cardiovascular disease [45] and as a marker of long life [46] because it is known to be influenced both by genetic and environmental factors [47] and is known to be moderated by a number of gene products. It is unlikely that heart disease causes elevated cholesterol and much more likely that the underlying risks for heart disease are manifest in hypercholesterolemia which in turn leads to abnormal cardiac function and eventual infarction. Implicit in endophenotype models of ADHD and other disease traits is the notion that underlying causative genetic variance influences certain traits (e.g., brain structure or cognitive function) which in turn are the causes of the disease phenotype. Various measures could serve as endophenotypes, but the prime candidates for endophenotypes in ADHD are the cognitive deficits that are readily detected in ADHD. There are many such deficits including deficits in various aspects of inhibitory control, working memory, sustained attention, processing speed and response variability. In the final analysis, the best endophenotype will be the one that proves to be most useful in genetic research by subgrouping affected individuals (those with and without a particular cognitive deficit) or as an alternative phenotype. Over the last few years, research into identifying optimal endophenotypes of ADHD has been intensifying.

Among the important criteria for a valid endophenotype is that the putative endophenotype is influenced by genetic factors and that these influences are common to those influences on ADHD itself. Family studies offer one way to test this hypothesis. If a putative endophenotype is under genetic influence, then unaffected relatives of affected individuals ought to manifest the trait $[48,49]$. There have been multiple studies demonstrating that apparently unaffected siblings and parents of individuals with ADHD have deficient response inhibition, slow and processing speed and several other traits [50]. Crosbie et al., studied response inhibition, latency and variability in relationship to ADHD traits in a general population sample of about 15,000 children and youth and observed clustering of ADHD and cognitive traits within families [51]. Twin studies afford another critical test of the same hypothesis. Schachar et al., found that several aspects of response inhibition were heritable and shared genetic risk with each other and with ADHD traits [52]. Others have observed that response time variability is under genetic control in ADHD [53].

On the horizon are studies in which brain structure and function as assessed using neuroimaging techniques of increasing sophistication are linked to increasingly more detailed mapping of individual genomes for identification of specific structure-function relationships in ADHD. Given the small size of the expected effects, it will be critical to pursue collaborative studies (see Enigma Consortium; http://enigma. loni.ucla.edu). Neuroimaging genetics deserves a review in its own right and will not be discussed here.

\section{Conclusions}

Genetic research strongly supports the role of genetic risk factors in ADHD. Yet, collectively current candidate gene, genome-wide and CNV studies explain relatively little of the apparent heritability of ADHD. Collectively, this "problem" is referred to as the "missing heritability" problem. It is not specific to ADHD genetics but applies to current findings in all complex traits. Clearly, the field is challenged to explain accounts for this missing heritability for which there are numerous possible reasons. Current genome-wide methods are unable to detect the effect of rare variants that collectively could account for a substantial amount of human disease but which are individually rare. Secondly, current array technologies do not adequately cover all possible sources of variation in the human genome. Thirdly, we have a very incomplete understanding of how various genes interact among themselves and with environmental risks in shaping a phenotype. Fourth, some genetic variants are known to affect phenotype only when inherited from a particular parent. Finally, epigenetic factors could well play a role in ADHD but are entirely independent of DNA sequence. Epigenetics refers to noncoding variation in the human genome that can affect gene expression and can be transmitted from one generation to the next. Epigenetics deserves its own discussion.

The emerging picture is one of substantial complexity similar to that found in other psychiatric disorders and many medical conditions. It is likely that genetic research will fractionate ADHD into various dimensions involving genetic risks that are partially unique and partially shared with other 
dimensions. These dimensions might involve the familiar inattention versus hyperactivity-impulsivity distinction but also might involve comorbid subtypes (e.g., ADHD with and without substance abuse disorder), [54], behaviors in different situations and at different points in development. Some genetic influences likely play a role in persistence into adulthood of childhood symptoms whereas other influences may come and go or affect the timing of critical aspects of brain or cognitive development. Current findings suggest that we will find both common variants and rare variants in ADHD with most variants being inherited but with others being do novo. We are on the verge of an entirely new generation of genetic research driven by the ability to sequence a person's entire genome for a reasonable cost although the analytic challenges will be formidable. To date, evidence for the environmental factors acting directly or indirectly via gene-environmental interaction is weak, but intensive study is ongoing, and it is reasonable to assume that such factors will be found. These efforts will require a far better understanding of the environments that are likely to interact with genetic risk. This type of research will require large samples as well because some environmental exposures might affect few individuals. The role of epigenetic mechanisms in the action of genetic risks in ADHD has barely been studied, but will be, no doubt, actively pursued. In addition to the above complexities, there is growing evidence that some causal genetic risks might increase likelihood of multiple psychiatric illnesses whereas others might be specific to ADHD or any other single disorder. Comparison of the genetic signatures of various disorders will probably become the standard of genetic research in the near future.

Perhaps the greatest current impediment to progress in ADHD is that of sample size. Efforts to build an ADHD genetics consortium have been quite successful. But larger samples are required. In the sample size game, ADHD lags well behind despite the fact that ADHD is the most highly prevalent of inherited mental illnesses and is well short of the point at which initial genome-wide significant findings tend to appear. We have to redouble our efforts at sample collection and ensure that ADHD receives the attention it deserves among funders and advocacy groups. ADHD genetics will also need to explore alternative research designs. For the most part, genetic studies have been based on case-control designs in which samples are collected from affected individuals and compared with unaffected controls. This strategy involves several inherent problems. First, criteria for ADHD are quite variable across sites and over time. Even though existing diagnostic strategies are appropriate for clinical purposes, variation in assessment and diagnostic approaches may introduce unhelpful "noise" into genetic analyses. The problem is compounded by inclusion of ADHD samples from around the world. It might be time for the adoption of research diagnostic criteria and dimensional measures (http://www.nimh.nih.gov/ research-priorities/rdoc/index.shtml) in addition to clinical criteria such as DSM 5. Second, it is clear that the distinction between cases and controls, even if reliably made across sites and over time, may reduce the power to discover causal genetic (or other) influences. If ADHD is the extreme of a normally distributed trait, then individuals who are just above whatever threshold one sets are not much different than those who are just below the threshold because causative variants can generate substantial variation even in supposedly normal individuals. This effect can be readily seen in population trait studies $[13 \bullet \bullet, 51]$. Quantitative trait measures in future genetic studies will allow distinctions to be made in both clinical and control samples. Given the high prevalence of ADHD-traits in the general population, many readily available genomic control samples could generate false negatives because of the inadvertent inclusion of controls with high levels of ADHD traits. Recent simulations indicate that if a disorder is prevalent in the population and individual SNPs account for substantial phenotypic variance, population samples assessed with trait measures may confer as much power as case-control studies at similar sample size. The advantage of population-based studies using quantitative traits, of course, is their feasibility.

A good argument can be made for the utility of nonbehavioral phenotypes (subphenotypes or endophenotypes) in the next wave of ADHD genetic research. If this is going to happen, it will be critical for ADHD geneticists to agree to a limited number of common measures. Otherwise, it will be impossible to achieve required sample size. Good candidates for the role of ADHD endophenotype must undergo rigorous testing prior to inclusion in genetic research if we are to avoid expensive and time-consuming mistakes.

Genetic methods are now very powerful and will soon be able to sequence an individual's entire genome. Variants of any kind will require careful replication and follow up research for understanding any potential causal role. There will be a need to study how the variant, if inherited, segregates with ADHD in family members. We will need to screen large numbers of individuals in the general population to establish a link between the CNV and the phenotype. Given what we now know about shared genetic risks across disorders, it will be critical to compare any genetic findings in ADHD with those in other disorders [40 $]$. Future studies of ADHD in relationship to other psychiatric disorders hold the promise of a redefinition of the boundaries between disorders, refined diagnosis and early detection of risk.

Genetic research carries critical implications for practitioners, educators and patients and quite possibly for the general public who shows enormous interest in genetics and its implications for their health. In some clinical situations, such as presence of ASD or intellectual deficiency, genetic arrays are already being run on a routine basis for clinical purposes. It may not be long before genetic arrays can be used in routine practice for other neurodevelopmental disorders such as 
ADHD. Even if genetic testing is not available as a medical test, it might not be long before ADHD patients begin to avail themselves of commercially available genetic testing. As a result, practitioners must be current with genetic findings and understand the risks carried by any particular genetic factor, the relative risks and benefits of genetic testing and the importance of communicating these risks to both affected individuals and their relatives (National Coalition for Health Professional Education in Genetics http://www.nchpeg.org/index.php?option= com_content\&view=article \&id=120\&Itemid=118). Practitioners much learn both what to tell patients and their families and how to communicate this information.

As genome-wide arrays become available in clinical practice, clinicians will need to prioritize which patients to send for testing. For the moment, priority should probably be given to patients with congenital anomalies, low intelligence, multiple medical problems, positive family history or dysmorphic features, because these seem to be the cases that are generating the highest yield of rare, potentially causal CNVs. Some of the genetic results may soon be useful for prediction of future disorder whereas other results may not be useful for prediction although these results might help us in the short run learn more about ADHD. Practitioners will need to be aware of the possibility of false positive (unanticipated) findings and the effects that genetic testing might have on treatment decisions, pregnancy, stigmatization and insurability. Recent research indicates that patients are overwhelmingly interested in receiving information about their genetic risks. Geneticallyinformed counseling for ADHD may soon become an important aspect of clinical care.

Acknowledgment Support provided by Canadian Institutes of Health Research (MOP 64277, 44070, 74699), Ontario Research Fund, and Ontario Brian Institute.

\section{Compliance with Ethics Guidelines}

Conflict of interest Russell Schachar declares that he has no conflict of interest.

Human and Animal Rights and Informed Consent This article does not contain any studies with human or animal subjects performed by any of the authors.

\section{References}

Papers of particular interest, published recently, have been highlighted as:

- Of importance

•• Of major importance

1. Vandenberg SG. Possible hereditary factors in minimal brain dysfunction. Ann N Y Acad Sci. 1973;205:223-30.
2. Omenn GS. Genetic issues in the syndrome of minimal brain dysfunction. Semin Psychiatr. 1973;5(1):5-17.

3. Willcutt EG. The prevalence of DSM-IV attention-deficit/hyperactivity disorder: a meta-analytic review. Neurother J Am Soc Exp NeuroTher. 2012;9(3):490-9.

4. Schachar R, Chen S, Crosbie J, Goos L, Ickowicz A, Charach A. Comparison of the predictive validity of hyperkinetic disorder and attention deficit hyperactivity disorder. J Can Acad Child Adolesc Psychiatr. 2007;16(2):90-100.

5. Bleck J, DeBate RD. Exploring the co-morbidity of attentiondeficit/hyperactivity disorder with eating disorders and disordered eating behaviors in a nationally representative community-based sample. Eat Behav. 2013;14(3):390-3.

6. Sinopoli KJ, Schachar R, Dennis M. Traumatic brain injury and secondary attention-deficit/hyperactivity disorder in children and adolescents: the effect of reward on inhibitory control. J Clin Exp Neuropsychol. 2011;33(7):805-19.

7. Ornstein TJ, Max JE, Schachar R, Dennis M, Barnes M, EwingCobbs L, et al. Response inhibition in children with and without ADHD after traumatic brain injury. J Neuropsychol. 2013;7(1):111.

8. Ficks CA, Lahey BB, Waldman ID. Does low birth weight share common genetic or environmental risk with childhood disruptive disorders? J Abnorm Psychol. 2013;122(3):842-53.

9. Collins FS. Reengineering translational science: the time is right. Sci Transl Med. 2011;3(90):90 cm17.

10. Association AP. Diagnostic and statistical manual of mental disorders Washington, DC: Author; 1994.

11. Association AP. Diagnostic and statistical manual of mental disorders. 5th ed. Arlington, VA: American Psychiatric Association Publishing; 2013.

12. Collins AL, Sullivan PF. Genome-wide association studies in psychiatry: what have we learned? Br J Psychiatr:J Ment Sci. 2013;202(1):1-4.

13.• Plomin R, Haworth CM, Davis OS. Common disorders are quantitative traits. Nat Rev Genet. 2009;10(12):872-8. This paper argues that a qualitative disorders can be interpreted simply as being the extreme of quantitative dimension if it is based on a polygenic mechanism. If this theory is correct, research should focus on quantitative traits - including the low and high ends of normal distributions and should avoid traditional case-control designs.

14. Akutagava-Martins GC, Salatino-Oliveira A, Kieling CC, Rohde LA, Hutz MH. Genetics of attention-deficit/hyperactivity disorder: current findings and future directions. Expert Rev Neurother. 2013;13(4):435-45.

15. Anney RJ, Lasky-Su J, O'Dushlaine C, Kenny E, Neale BM, Mulligan A, et al. Conduct disorder and ADHD: evaluation of conduct problems as a categorical and quantitative trait in the international multicentre ADHD genetics study. Am J Med Gen Part B, Neuropsychiatr Genet: Off Publ Int Soc Psychiatr Genet. 2008;147B(8):1369-78.

16. Biederman J, Faraone SV, Petty C, Martelon M, Woodworth KY, Wozniak J. Further evidence that pediatric-onset bipolar disorder comorbid with ADHD represents a distinct subtype: results from a large controlled family study. J Psychiatr Res. 2013;47(1):15-22.

17. Rommelse NN, Franke B, Geurts HM, Hartman CA, Buitelaar JK. Shared heritability of attention-deficit/hyperactivity disorder and autism spectrum disorder. Eur Child Adolesc Psychiatr. 2010;19(3):281-95.

18. Biederman J, Pettye CR, Hammerness P, Woodworth KY, Faraon SV. Examining the nature of the association between attentiondeficit hyperactivity disorder and nicotine dependence: a familial risk analysis. Can J Psychiatr Revue Canadienne de Psychiatrie. 2013;58(3):177-83.

19. Faraone SV, Mick E. Molecular genetics of attention deficit hyperactivity disorder. Psychiatr Clin N Am. 2010;33(1):159-80. 
20. Visscher PM, Hill WG, Wray NR. Heritability in the genomics eraconcepts and misconceptions. Nat Rev Genet. 2008;9(4):255-66. This paper is an approachable discussion of common misconceptions about the genetics of complex disorders.

21.• Sullivan PF, Daly MJ, O'Donovan M. Genetic architectures of psychiatric disorders: the emerging picture and its implications. Nat Rev Genet. 2012;13(8):537-51. A review of the progress that has been made in the genetics of various complex disorders which places neuropsychiatric genetics in this broader context.

22. Chang Z, Lichtenstein P, Asherson PJ, Larsson H. Developmental twin study of attention problems: high heritabilities throughout development. JAMA Psychiatr. 2013;70(3):311-8.

23. Greven CU, Asherson P, Rijsdijk FV, Plomin R. A longitudinal twin study on the association between inattentive and hyperactiveimpulsive ADHD symptoms. J Abnorm Child Psycholog. 2011;39(5):623-32.

24. Ronald A, Simonoff E, Kuntsi J, Asherson P, Plomin R. Evidence for overlapping genetic influences on autistic and ADHD behaviours in a community twin sample. J Child Psychol Psychiatr Allied Discip. 2008;49(5):535-42.

25. Lichtenstein P, Carlstrom E, Rastam M, Gillberg C, Anckarsater H. The genetics of autism spectrum disorders and related neuropsychiatric disorders in childhood. Am J Psychiatr. 2010;167(11): 1357-63.

26. Ronald A, Larsson H, Anckarsater H, Lichtenstein P. A twin study of autism symptoms in Sweden. Mol Psychiatr. 2011;16(10):1039-47.

27. Faraone SV, Biederman J, Wozniak J. Examining the comorbidity between attention deficit hyperactivity disorder and bipolar I disorder: a meta-analysis of family genetic studies. Am J Psychiatr. 2012;169(12):1256-66

28. Willcutt EG, Pennington BF, DeFries JC. Twin study of the etiology of comorbidity between reading disability and attention-deficit/ hyperactivity disorder. Am J Med Genet. 2000;96(3):293-301.

29. Greven CU, Harlaar N, Dale PS, Plomin R. Genetic Overlap between ADHD Symptoms and Reading is largely Driven by Inattentiveness rather than Hyperactivity-Impulsivity. J Can Acad Child Adolesc Psychiatr. 2011;20(1):6-14

30. Cole J, Ball HA, Martin NC, Scourfield J, McGuffin P. Genetic overlap between measures of hyperactivity/inattention and mood in children and adolescents. J Am Acad Child Adolesc Psychiatry. 2009;48(11):1094-101.

31. Thapar A, Harrington R, McGuffin P. Examining the comorbidity of ADHD-related behaviours and conduct problems using a twin study design. Br J Psychiatr: J Ment Sci. 2001;179:224-9.

32. Wong CC, Mill J, Fernandes C. Drugs and addiction: an introduction to epigenetics. Addiction. 2011;106(3):480-9.

33. Gizer IR, Ficks C, Waldman ID. Candidate gene studies of ADHD: a meta-analytic review. Human Genet. 2009;126(1):51-90.

34. Bosker FJ, Hartman CA, Nolte IM, Prins BP, Terpstra P, Posthuma $\mathrm{D}$, et al. Poor replication of candidate genes for major depressive disorder using genome-wide association data. Mol Psychiatr. 2011;16(5):516-32.

35. Sullivan PF. Spurious genetic associations. Biol Psychiatr. 2007;61(10):1121-6.

$36 . \bullet$ Cross-Disorder Group of the Psychiatric Genomics C, Lee SH, Ripke S, Neale BM, Faraone SV, Purcell SM, et al. Genetic relationship between five psychiatric disorders estimated from genome-wide SNPs. Nat Genet. 2013;45(9):984-94. The largest genetic study of neuropsychiatric disorders that has been done to date. This study provides several novel candidate loci for follow up and shows that even though various mental disorders can be distinguished clinically, they may share genetic mechanisms to some extent.

37. Neale BM, Medland SE, Ripke S, Asherson P, Franke B, Lesch KP, et al. Meta-analysis of genome-wide association studies of attention-deficit/hyperactivity disorder. J Am Acad Child Adolesc Psychiatry. 2010;49(9):884-97.
38. Zhou K, Dempfle A, Arcos-Burgos M, Bakker SC, Banaschewski $\mathrm{T}$, Biederman J, et al. Meta-analysis of genome-wide linkage scans of attention deficit hyperactivity disorder. Am J Med Genet Part B, Neuropsychiatr Genet: Off Publ Int Soc Psychiatric Genet. 2008;147B(8):1392-8.

39. Poelmans G, Pauls DL, Buitelaar JK, Franke B. Integrated genomewide association study findings: identification of a neurodevelopmental network for attention deficit hyperactivity disorder. Am J Psychiatr. 2011;168(4):365-77.

40. Lionel AC, Crosbie J, Barbosa N, Goodale T, Thiruvahindrapuram B, Rickaby J, et al. Rare copy number variation discovery and cross-disorder comparisons identify risk genes for ADHD. Sci Transl Med. 2011;3(95):95ra75. This study uncovers CNVs in 9\% of ADHD cases and provides clinical vignettes that link genetic findings to clinical care. Moreover, this study shows that $50 \%$ of $A D H D C N V$ s can be found in individuals with ASD.

41. Stam AJ, Schothorst PF, Vorstman JA, Staal WG. The genetic overlap of attention deficit hyperactivity disorder and autistic spectrum disorder. Appl Clin Genet. 2013;2:7-13.

42. Williams NM, Franke B, Mick E, Anney RJ, Freitag CM, Gill M, et al. Genome-wide analysis of copy number variants in attention deficit hyperactivity disorder: the role of rare variants and duplications at 15q13.3. Am J Psychiatr. 2012;169(2):195-204.

43. Thapar A, Rice F, Hay D, Boivin J, Langley K, van den Bree M, et al. Prenatal smoking might not cause attention-deficithyperactivity disorder: evidence from a novel design. Biol Psychiatr. 2009;66(8):722-7.

44. Ficks CA, Waldman ID. Gene-environment interactions in attention-deficit/hyperactivity disorder. Curr Psychiatr Rep. 2009;11(5):387-92.

45. Morabia A, Cayanis E, Costanza MC, Ross BM, Flaherty MS, Alvin GB, et al. Association of extreme blood lipid profile phenotypic variation with 11 reverse cholesterol transport genes and 10 non-genetic cardiovascular disease risk factors. Human Mol Genet. 2003;12(21):2733-43.

46. Matteini AM, Fallin MD, Kammerer CM, Schupf N, Yashin AI, Christensen K, et al. Heritability estimates of endophenotypes of long and health life: the Long Life Family Study. J Gerontol Series A, Biol Sci Med Sci. 2010;65(12):1375-9.

47. Weissglas-Volkov D, Pajukanta P. Genetic causes of high and low serum HDL-cholesterol. J Lipid Res. 2010;51(8):2032-57.

48. Gottesman II, Gould TD. The endophenotype concept in psychiatry: etymology and strategic intentions. Am J Psychiatr. 2003;160(4):636- 45.

49. Crosbie J, Perusse D, Barr CL, Schachar RJ. Validating psychiatric endophenotypes: inhibitory control and attention deficit hyperactivity disorder. Neurosci Biobehav Rev. 2008;32(1):40-55.

50. Schachar RJ, Crosbie J, Barr CL, Ornstein TJ, Kennedy J, Malone $\mathrm{M}$, et al. Inhibition of motor responses in siblings concordant and discordant for attention deficit hyperactivity disorder. Am J Psychiatr. 2005;162(6):1076-82.

51. Crosbie J, Arnold P, Paterson A, Swanson J, Dupuis A, Li X, et al. Response inhibition and ADHD traits: correlates and heritability in a community sample. J Abnorm Child Psychol. 2013;41(3):497-507.

52. Schachar RJ, Forget-Dubois N, Dionne G, Boivin M, Robaey P. Heritability of response inhibition in children. J Int Neuropsychol Soc: JINS. 2011;17(2):238-47.

53. McLoughlin G, Palmer JA, Rijsdijk F, Makeig S. Genetic Overlap between Evoked Frontocentral Theta-Band Phase Variability, Reaction Time Variability, and Attention-Deficit/Hyperactivity Disorder Symptoms in a Twin Study. Biological psychiatry. 2013.

54. Sanchez-Mora C, Ramos-Quiroga JA, Garcia-Martinez I, Fernandez N, Bosch R, Richarte V, et al. Evaluation of single nucleotide polymorphisms in the miR-183-96-182 cluster in adulthood attentiondeficit and hyperactivity disorder (ADHD) and substance use disorders (SUDs). European neuropsychopharmacology : the journal of the European College of Neuropsychopharmacology. 2013. 